\title{
Influence of Sulfate Ions on the Atomic-Scale Structure of $\beta$-FeOOH
}

\author{
Sang-Koo Kwon ${ }^{1}$, Shigeru Suzuki ${ }^{1, *}$, Masatoshi Saito ${ }^{2}$, Takayuki Kamimura ${ }^{3}$, \\ Hideaki Miyuki ${ }^{3}$ and Yoshio Waseda ${ }^{1}$ \\ ${ }^{1}$ Institute of Multidisciplinary Research for Advanced Materials, Tohoku University, Sendai 980-8577, Japan \\ ${ }^{2}$ School of Health Sciences, Faculty of Medicine, Niigata University, Niigata 951-8518, Japan \\ ${ }^{3}$ Corporate Research \& Development Laboratories, Sumitomo Metal Industries Ltd., Amagasaki 660-0891, Japan
}

Ordinary X-ray diffraction measurements by Mo K $\alpha$ radiation and anomalous X-ray scattering (AXS) measurements by synchrotron radiation below Fe $\mathrm{K}$ absorption edge were used for characterizing the atomic-scale structure of $\beta$-FeOOH particles with and without sulfate ions. Fourier-transform infrared spectroscopy (FT-IR) was also used for analyzing a change of the bonding structure in the $\beta$-FeOOH particles by addition of sulfate ions. The realistic atomic-scale structures of the $\beta$-FeOOH particles with and without sulfate ions were estimated by fitting both the ordinary and environmental interference functions with model calculation using the reverse Monte Carlo (RMC) simulation technique. The results showed that the linkages of $\mathrm{FeO}_{6}$ octahedral structural units in the $\beta$-FeOOH particles are distorted by addition of sulfate ions, although the structure of the $\beta$-FeOOH particles with and without sulfate ions is fundamentally similar to the ideal $\beta$-FeOOH structure. The structural distortion in the $\beta$-FeOOH particles with sulfate ions is likely to arise from the incorporation of sulfate ions. The atomic-scale structures visualized for these $\beta$-FeOOH particles were discussed coupled with the bonding structure observed in FT-IR.

(Received May 27, 2005; Accepted July 1, 2005; Published September 15, 2005)

Keywords: $\beta$-FeOOH, sulfate ions, X-ray diffraction, anomalous X-ray scattering, Reverse Monte Carlo simulation, atomic-scale structure

\section{Introduction}

Corrosion products of iron consist mainly of ferric hydroxides, oxyhydroxides, oxides, which are formed through anodic and cathodic reactions on the surface of iron or steel under various environments. ${ }^{1,2)}$ These components of corrosion products are determined by the amounts of chemical species, such as ferric and ferrous ions dissolved from the iron surface, corrosion conditions and so on. Practically, ferric oxyhydroxides are main components of the corrosion products, and they are known to be formed via olation and oxolation of the $\mathrm{FeO}_{6}$ units in water and finally dehydrated and/or dehydroxylated to ferric oxide. ${ }^{3-5)}$ The structures of ferric oxyhydroxides are fundamentally described by the linkages of $\mathrm{FeO}_{6}$ octahedral units and these octahedral units are linked by corners, edges, faces or combinations of these linkages to form different structural arrays. ${ }^{2,3)}$ The $\mathrm{FeO}_{6}$ octahedral structural units are maintained even in the amorphous state of ferric hydroxide $\left(\mathrm{Fe}(\mathrm{OH})_{3}\right),{ }^{6)}$ and the linkages of the $\mathrm{FeO}_{6}$ octahedral structural units are likely to be correlated with the morphology and stability of ferric oxyhydroxides. ${ }^{7-9)}$ Among ferric oxyhydroxides, the formation of $\beta$-FeOOH seems to be related to a process of atmospheric corrosion of steel, because $\beta$-FeOOH contains chloride ions. ${ }^{1,2)}$ Recently, the effect of cations and anions on the formation of $\beta$-FeOOH particles has been focused, since several elements are strongly influence the formation and structure of $\beta$-FeOOH. ${ }^{10-14)}$ However, an influence of anions on the atomic-scale structure of $\beta-\mathrm{FeOOH}$ has not been understood yet.

Although the extended X-ray absorption fine structure (EXAFS) method has been used for characterizing the local structure of corrosion products, ${ }^{15-17)}$ information obtained by EXAFS appears to be too short range to discuss the atomicscale structure consisting of the $\mathrm{FeO}_{6}$ octahedral structural

*Corresponding author, E-mail: ssuzuki@tagen.tohoku.ac.jp units. ${ }^{18-20)}$ Although other structural analysis methods provide useful information, they do not necessarily cover whole characteristic features of the structure of corrosion products. ${ }^{21-25)}$ Recently, the quantitative X-ray structural analysis coupled with anomalous X-ray scattering (AXS) and the reverse Monte Carlo (RMC) simulation has been successfully used for characterizing the atomic-scale structures of materials in various states such as liquids, glasses and aperiodic systems. ${ }^{20,26-28)}$ This method has been utilized for analyzing the atomic-scale structure of corrosion products formed on the surface of several iron-based alloys. ${ }^{7-9)}$ The results showed that the linkages of $\mathrm{FeO}_{6}$ octahedral structural units were considerably influenced by incorporation of cations and anions during the formation of corrosion products. AXS measurements are applied for estimating the environmental structure around specific cations, because cations are generally of metallic elements, of which the Xrays scattering factor is relatively large.$^{8)}$ On the other hand, anions such as carbonate and sulfate are composed of relatively light elements, by which X-rays are hardly scattered. Therefore, a combination of AXS measurements with other spectroscopic methods is effective in analysis of the atomic-scale structure of corrosion products containing anions. ${ }^{9)}$

Sulfate ions are known to play an important role in controlling the morphology of monodispersed hematite ( $\alpha$ $\mathrm{Fe}_{2} \mathrm{O}_{3}$ ) particles formed in aqueous solution. ${ }^{29-32)}$ The shape of $\alpha-\mathrm{Fe}_{2} \mathrm{O}_{3}$ particles can be controlled by the adsorption of sulfate ions on the surface parallel to the $c$-axis of $\alpha-\mathrm{Fe}_{2} \mathrm{O}_{3}$. Such adsorption of sulfate ions is considered to suppress the crystal growth perpendicular to the $c$-axis, resulting in the anisotropic crystal growth in $\alpha-\mathrm{Fe}_{2} \mathrm{O}_{3}$ synthesis. ${ }^{33-35)}$ In addition, sulfate ions appear to give a significant influence on the conversion process from $\mathrm{Fe}(\mathrm{OH})_{3}$ to $\beta$ - $\mathrm{FeOOH}$ and $\alpha$ $\mathrm{Fe}_{2} \mathrm{O}_{3}$ particles. ${ }^{12)}$ Also, sulfate ions are likely to influence the structure of corrosion products, since sulfate ions are sometimes contained in corrosion atmosphere. 
This prompts us to study the influence of sulfate ions on the atomic-scale structure of $\beta$-FeOOH particles synthesized in aqueous solution. In this study, the ordinary X-ray diffraction and AXS measurements below Fe $\mathrm{K}$ absorption edge were carried out for analyzing the atomic-scale structures of $\beta$ $\mathrm{FeOOH}$ with and without sulfate ions. The realistic atomicscale structures of these $\beta$-FeOOH samples were estimated by fitting the interference functions by the RMC simulation method to the experimental results. The atomic-scale structures were discussed coupled with the results obtained by Fourier-transform infrared spectroscopy (FT-IR).

\section{Experimental}

\subsection{Sample preparation}

Pure $\beta$-FeOOH particles with colloidal state were synthesized by hydrolysis of $0.1 \mathrm{M}$ ferric chloride $\left(\mathrm{FeCl}_{3}\right)$ solution through aging at $373 \mathrm{~K}$. Sulfate ions were added to the initial $\mathrm{FeCl}_{3}$ solution by $30 \mathrm{~mol} \%$ to ferric ions to prepare $\beta$-FeOOH particles containing sulfate ions. These $\beta$-FeOOH particles without and with sulfate ions are hereafter referred to as $\beta$ $\mathrm{FeOOH}$ and $\beta-30 \mathrm{~S}$, respectively. Electron microprobe analysis of $\beta-\mathrm{FeOOH}$ showed that the molar ratio of chorine to iron was 0.17 in it, which are comparable to the ratio for the ideal $\beta$-FeOOH. ${ }^{2)}$ On the other hand, the molar ratios of chorine and sulfur to iron in $\beta$-30S were approximately 0.08 and 0.09 , respectively. This suggests that sulfate ions are replaced with about $53 \%$ of chloride ions in $\beta$-30S.

\subsection{Measurements}

Ordinary X-ray diffraction measurements were carried out by an in-house X-ray diffraction apparatus Rigaku RINT2200 , in which Mo K $\alpha$ radiation of $17.447 \mathrm{keV}(50 \mathrm{kV}$, $30 \mathrm{~mA}$ ) was used. The X-ray scattering intensities were measured in a wide range of wave vector, in order to obtain the atomic-scale structure with reliable resolution. AXS measurements were conducted to obtain the environmental interference function for iron in the $\beta$-FeOOH particles. The beam line used for AXS measurements below Fe K absorption edge was in BL-7C of Photon Factory, High Energy Accelerator Research Organization at Tsukuba in Japan. Atomic scattering factor is expressed by the normal atomic scattering factor and the real and imaginary components of the anomalous dispersion term, $f^{\prime}$ and $f^{\prime \prime} .^{20)}$ The energies of incident radiations for AXS measurements for Fe were 7.086 and $6.811 \mathrm{keV}$, which correspond to 25 and $300 \mathrm{eV}$ below the Fe K absorption edge, respectively.

The fundamental local octahedral units of $\mathrm{FeO}_{6}$ and their linkages in the $\beta$-FeOOH particles were described using the partial distribution functions obtained by the RMC simulation technique. ${ }^{7,26-28)}$ The RMC routine is essentially identical to that used in our previous works. ${ }^{8)}$ The super-cell of $\beta$ $\mathrm{FeOOH}$ was established with a dimension of $4.224 \times$ $4.192 \times 4.242 \mathrm{~nm}^{3}$ in this RMC simulation to secure sufficient atoms in crystal for the computational accuracy. The simulation was conducted with an initial configuration of 5824 atoms (1792 for iron, 3584 for oxygen, 448 for chloride) positioned in the super-cell. The usual periodic boundary conditions, in which the cell is surrounded by the images of itself, were applied, and the interference functions were

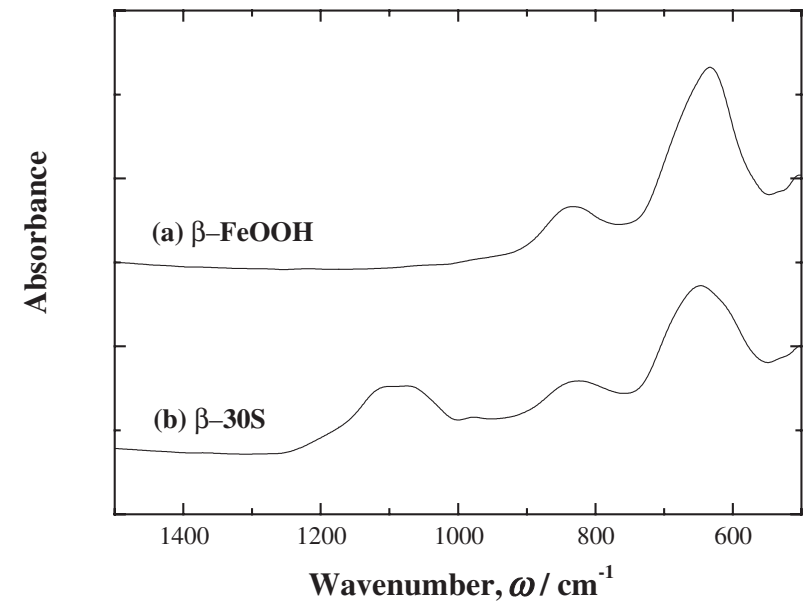

Fig. 1 Infrared absorption spectra of (a) $\beta$-FeOOH and (b) $\beta-30 \mathrm{~S}$.

calculated to obtain the quantitative structural information related with nearest-neighboring correlations. Detailed methods for these AXS measurements and analyses are described elsewhere. $^{20)}$

A change of the bonding structure in $\beta$-FeOOH particles by addition of sulfate ions was analyzed by Fourier-transform infrared spectroscopy (FT-IR), in order to investigate the influence of sulfate ions on the atomic-scale structure of $\beta$ $\mathrm{FeOOH}$.

\section{Results and Discussion}

\subsection{Infrared spectroscopy}

Figure 1 shows IR spectra of $\beta$-FeOOH and $\beta$-30S prepared in this work, which were measured a range of $500-1500 \mathrm{~cm}^{-1}$. Two main absorption peaks are observed at about 630 and $830 \mathrm{~cm}^{-1}$ in the spectrum of $\beta$-FeOOH. On the other hand, absorption bands appeared at about $1090 \mathrm{~cm}^{-1}$ in $\beta$-30S, in addition to the main absorption peaks. These absorption bands are considered to be associated with sulfate ions in the tunnels of the $\beta$-FeOOH structure and sulfate ions adsorbed on the particles, ${ }^{1,36)}$ although the precise bonding structure is unclear yet.

\subsection{Ordinary X-ray diffractions and anomalous X-ray scattering profiles}

Figure 2 shows the ordinary X-ray diffraction profiles of $\beta$-FeOOH and $\beta$-30S, which were measured by the in-house $\mathrm{X}$-ray diffraction apparatus. The reference lines for $\beta$ $\mathrm{FeOOH}$, given in the JCPDS database, are also denoted in Fig. 2. The results indicate that the structure of these samples is fundamentally the $\beta$-FeOOH structure and other phases are not contained. However, the X-ray diffraction profile of $\beta$ $30 \mathrm{~S}$ indicates that the atomic-scale structure of $\beta-30 \mathrm{~S}$ is distorted by the incorporation of sulfate ions. Although the incorporated sulfate ions may occupy some site in the structure of $\beta$-30S or adsorb on $\beta$-30S particles, techniques for obtaining such structural information appear to be limited. Nevertheless, the environmental structural analysis around iron as well as the average structural analysis of these samples may be available. Therefore, AXS measurements were carried out for studying a difference of the atomic-scale 


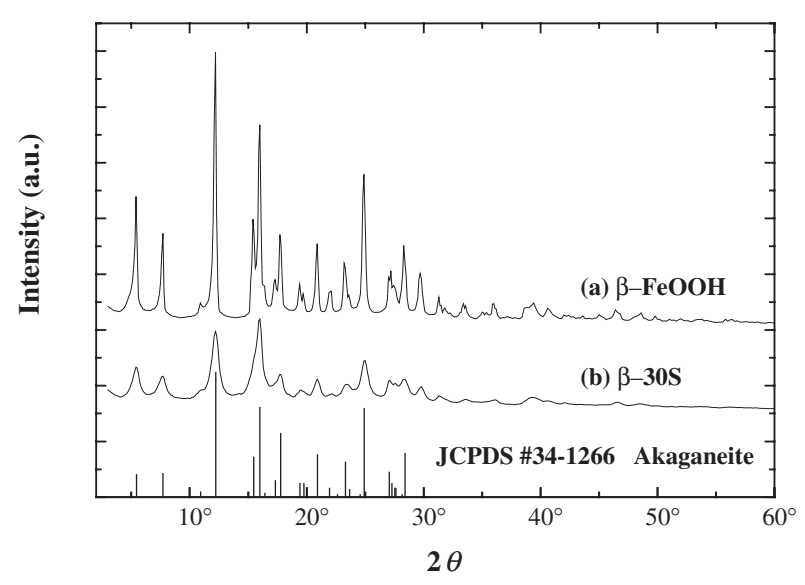

Fig. 2 Ordinary X-ray diffraction profiles for $\beta$-FeOOH and $\beta$-30S obtained by in-house X-ray diffraction apparatus with Mo $\mathrm{K} \alpha$ radiation. The reference lines for $\beta$-FeOOH given in the JCPDS database are also denoted.

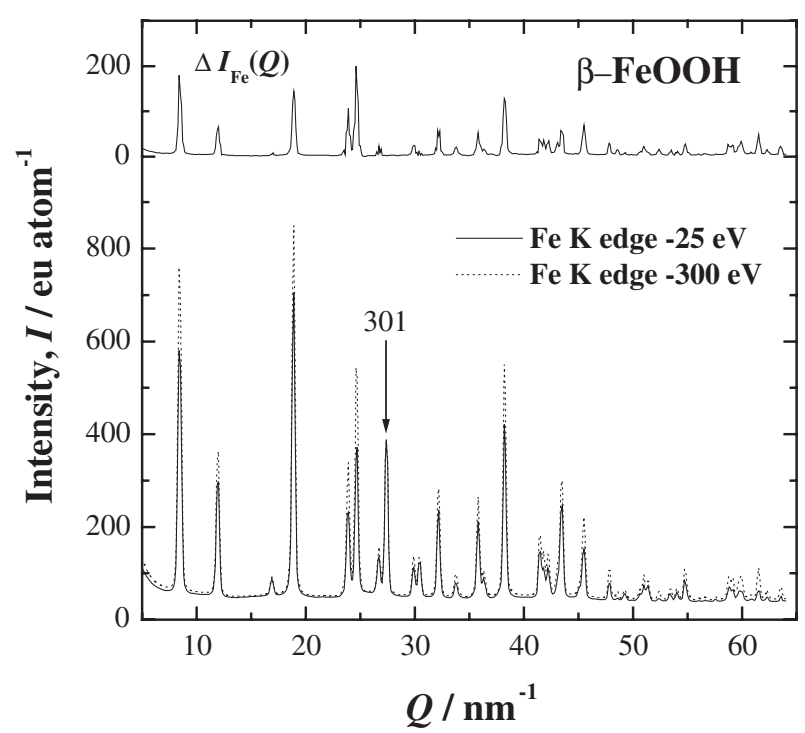

Fig. 3 Differential intensity profile (top) of $\beta$-FeOOH obtained from the intensity profiles (bottom) measured at incident energies of 7.086 and $6.811 \mathrm{keV}$, which correspond to the energies 25 and $300 \mathrm{eV}$ below the Fe K absorption edge, respectively.

structure between $\beta$-FeOOH and $\beta$-30S.

The anomalous X-ray scattering profiles for $\beta$-FeOOH, which were measured at incident energies 25 and $300 \mathrm{eV}$ below the Fe $\mathrm{K}$ absorption edge, are shown in Fig. 3. The diffraction intensities at $-300 \mathrm{eV}$ of $\mathrm{Fe} \mathrm{K}$ absorption edge appear to be higher than those of $-25 \mathrm{eV}$ of Fe $\mathrm{K}$ absorption edge. A differential X-ray intensity profile calculated from the two intensity profiles was given at the top of Fig. 3, which indicates the structural information around iron in $\beta-\mathrm{FeOOH}$. The differential intensity profile was obtained from the energy dependence of the anomalous dispersion factor in the vicinity of $\mathrm{Fe} \mathrm{K}$ absorption edge. It would be noteworthy that the diffraction peak for 301 planes in $\beta$-FeOOH disappears in the differential intensity profile, whereas this peak is observed in both scattering intensity profiles measured at incident energies 25 and $300 \mathrm{eV}$ below the $\mathrm{Fe} \mathrm{K}$ absorption

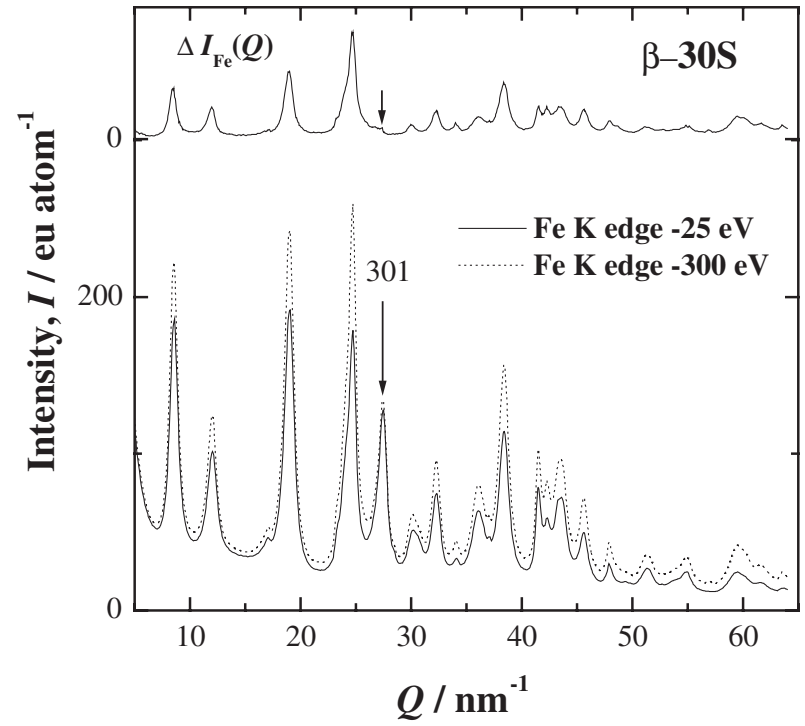

Fig. 4 Differential intensity profile (top) of $\beta$-30S obtained from the intensity profiles (bottom) measured at incident energies of 7.086 and $6.811 \mathrm{keV}$, which correspond to the energies 25 and $300 \mathrm{eV}$ below the Fe K absorption edge, respectively.

edge. This is because the peak originates from arrays of oxygen in crystal, indicating that the differential intensity profile enables us to provide the environmental structural information around iron in $\beta-\mathrm{FeOOH}$.

Figure 4 shows the anomalous X-ray scattering profiles for $\beta$-30S, which were measured at incident energies 25 and $300 \mathrm{eV}$ below the Fe $\mathrm{K}$ absorption edge. The differential Xray intensity profile is given at the top of Fig. 4. The diffraction peaks for 301 planes by oxygen, which were not observed in the differential intensity profile of $\beta$-FeOOH, were slightly detected. Although the origin of the difference between these two differential profiles in $\beta$-FeOOH and $\beta$-30 cannot be certainly identified at the present time, it may be related with heterogeneous distribution of cations and anions such as chloride and sulfate ions in the particles. Thus, the results obtained by AXS measurements provide information of environmental structures for iron in $\beta-\mathrm{FeOOH}$ and $\beta-30 \mathrm{~S}$, which is not available from data obtained by ordinary $\mathrm{X}$-ray diffraction alone.

\subsection{Experimental and calculated interference functions}

The experimental ordinary interference function $Q i(Q)$ and environmental interference function $Q \Delta i_{\mathrm{Fe}}(Q)$ for iron of $\beta$ $\mathrm{FeOOH}$ in the absence of sulfate ions are shown as dots in Figs. 5(a) and (b). These experimental interference functions were calculated based on the ordinary X-ray diffraction profiles and the anomalous X-ray scattering profiles given in Figs. 2 and 3, respectively. In a similar manner, the ordinary interference function $Q i(Q)$ and the environmental interference function $Q \Delta i_{\mathrm{Fe}}(Q)$ for iron of $\beta$-30S were obtained from the experimental ordinary and anomalous X-ray profiles, as shown in Figs. 6(a) and (b).

The realistic atomic-scale arrangements in $\beta$-FeOOH and $\beta$-30S were estimated by fitting these interference functions transformed from the experimental data with a model using the reverse Monte Carlo (RMC) simulation technique. The 


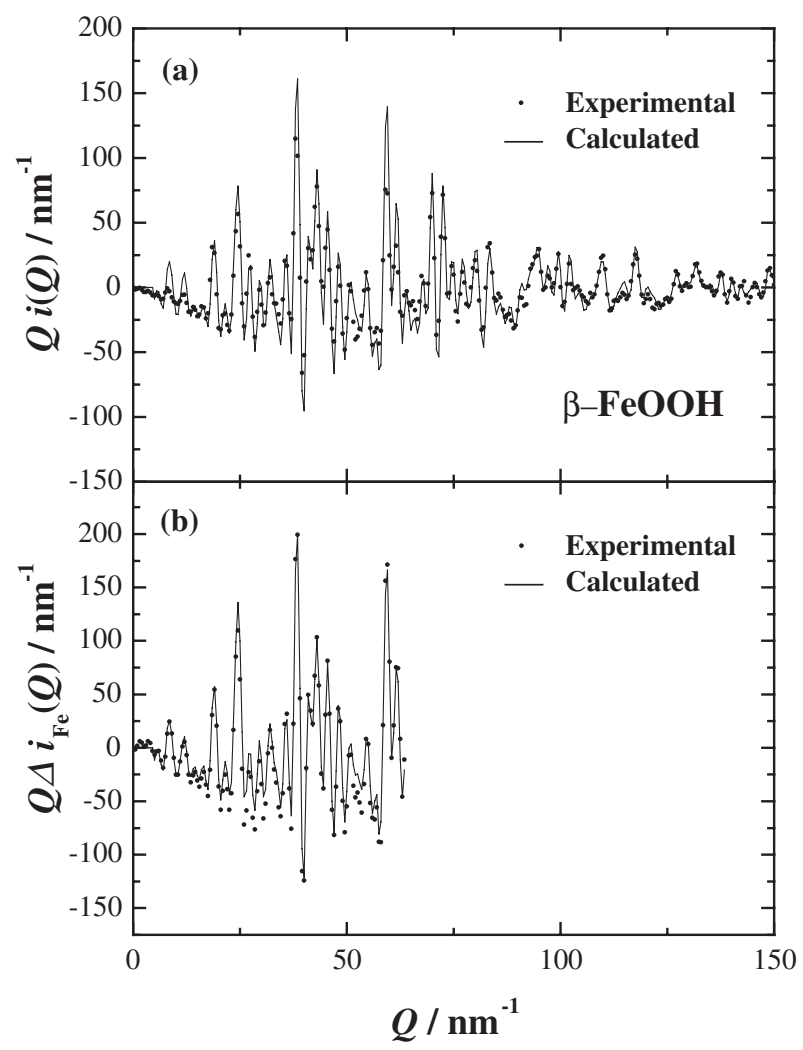

Fig. 5 The ordinary interference function $Q i(Q)$ and environmental interference functions $Q \Delta i_{\mathrm{Fe}}(Q)$ for iron in $\beta$-FeOOH. Dots and solid lines denote the experimental data and values calculated by RMC simulation, respectively.

interference functions computed by the RMC method were compared with the experimental interference functions shown in Figs. 6(a) and (b), in order to analyze fundamental linkages of $\mathrm{FeO}_{6}$ structural units and atomic-scale structures of $\beta$-FeOOH and $\beta$-30S. New atomic configurations in the super-cell were generated by the random movement of atom positions, and the interference functions were calculated by repetition of acceptance and rejection compared with previous configuration data. When the difference between experimental results and calculated values is smaller than that of previous configuration, the new configuration is accepted. This iterative calculation was done with sufficient time until a reasonable convergence was obtained by statistical optimization. The results of RMC simulation are given as solid lines in Figs. 5 and 6. The computed interference functions are in good agreement with the experimental results, although some deviations seem to arise from relatively simple assumptions in RMC simulation.

\subsection{Partial pair distribution functions estimated by RMC}

Figure 7 shows the pair distribution functions of $\beta-\mathrm{FeOOH}$ and $\beta-30 \mathrm{~S}$, which were estimated from new atomic configurations for $\beta$-FeOOH and $\beta$-30S by statistical optimization between experimental and calculated interference functions through the RMC simulation technique. The partial pair distribution functions of $\mathrm{Fe}-\mathrm{O}, \mathrm{Fe}-\mathrm{Fe}$ and $\mathrm{O}-\mathrm{O}$ pairs in $\beta$ $\mathrm{FeOOH}$ and $\beta$-30S are shown in Figs. 7(a), (b) and (c), respectively. The first peak of $g_{\mathrm{Fe}-\mathrm{O}}$ around $0.2 \mathrm{~nm}$ in $\beta-30 \mathrm{~S}$ is

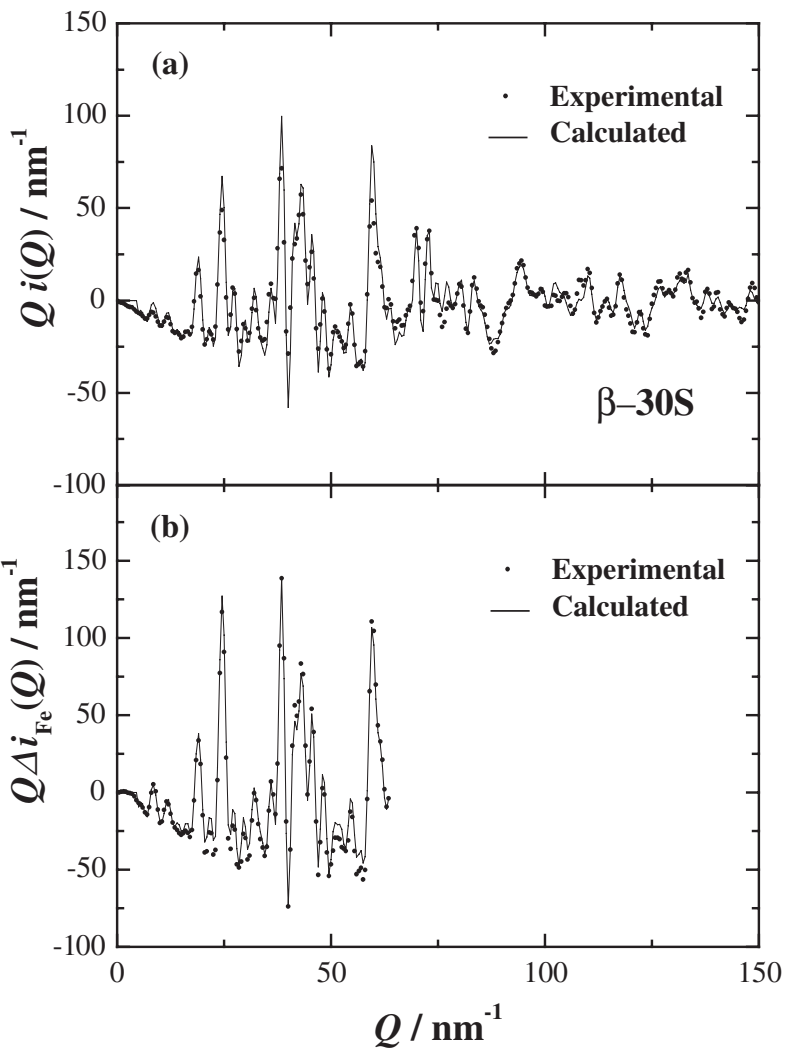

Fig. 6 The ordinary interference function $Q i(Q)$ and environmental interference functions $Q \Delta i_{\mathrm{Fe}}(Q)$ for iron in $\beta$-30S. Dots and solid lines denote the experimental data and values calculated by RMC simulation, respectively.
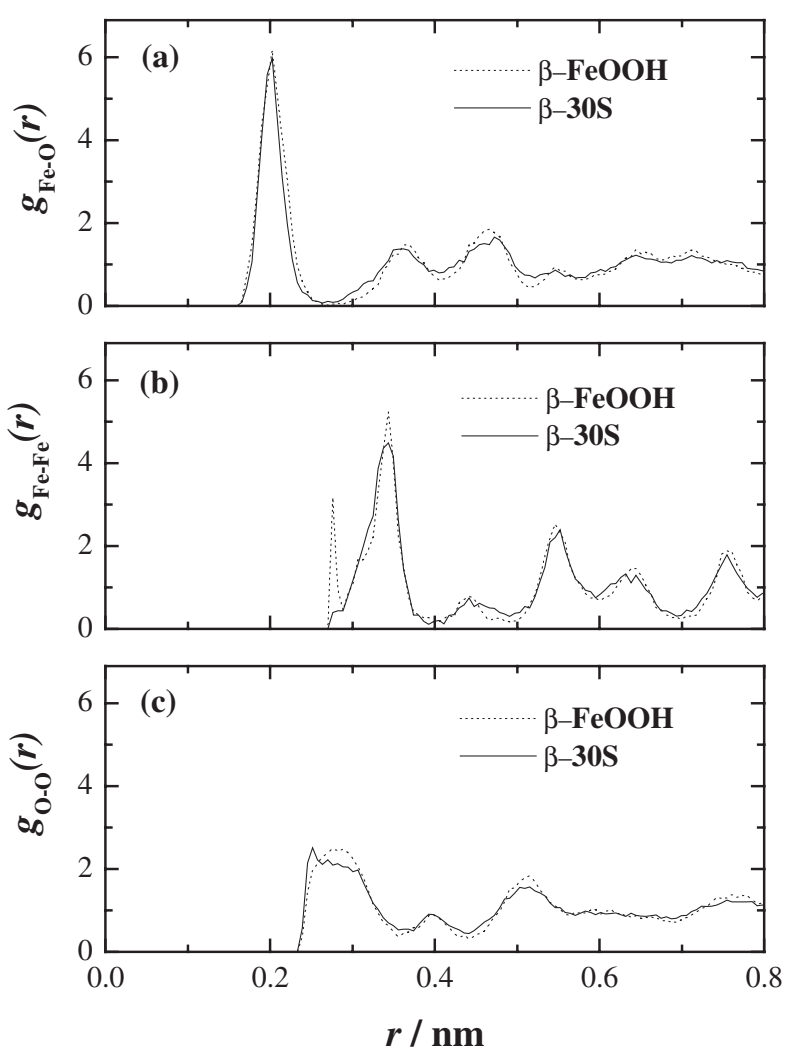

Fig. 7 Partial pair distribution functions of (a) $\mathrm{Fe}-\mathrm{O}$, (b) $\mathrm{Fe}-\mathrm{Fe}$ and (c) $\mathrm{O}-$ $\mathrm{O}$ pairs in $\beta$-FeOOH and $\beta$-30S estimated by RMC simulation. 
similar to that of $\beta$-FeOOH, as shown in Fig. 7(a). This suggests that the fundamental structure of $\mathrm{FeO}_{6}$ octahedral unit does not seem to be severely influenced by addition of sulfate ions. However, the peaks at about 0.35 and $0.45 \mathrm{~nm}$ in $g_{\mathrm{Fe}-\mathrm{O}}$ for $\beta$-30S are broader than that for $\beta$-FeOOH, and slightly shifted. This indicates that the iron-oxygen neighboring correlation in $\beta$-30S is widely distributed in the crystal. The pair distribution functions of $\mathrm{Fe}-\mathrm{Fe}, g_{\mathrm{Fe}-\mathrm{Fe}}$, for $\beta$ $30 \mathrm{~S}$ are quite different from that of $\beta-\mathrm{FeOOH}$, as shown in Fig. 7(b). The profile around the first peak in $g_{\mathrm{Fe}-\mathrm{Fe}}$ for $\beta-30 \mathrm{~S}$ is considerably deviated from that for $\beta$-FeOOH. Other distribution profiles of $g_{\mathrm{Fe}-\mathrm{Fe}}$ for $\beta$-30S are broader than those of $\beta-\mathrm{FeOOH}$, indicating that the atomic-scale structure is significantly changed by addition of sulfate ions in $\beta$-30S. The pair distribution functions of $\mathrm{O}-\mathrm{O}, g_{\mathrm{O}-\mathrm{O}}$, for $\beta$-30S are also broader than those in $\beta-\mathrm{FeOOH}$, as shown in Fig. 9(c). Thus, the whole profiles of the pair distribution functions for $\beta$-30S evidently show that the linkages of $\mathrm{FeO}_{6}$ octahedral structure units, which are associated with the middle-range order, are considerably distorted by the sulfate ions in $\beta$ $\mathrm{FeOOH}$.

The ideal structure of $\beta-\mathrm{FeOOH}$ is shown in Fig. 8(a), in which chloride ions are not shown for simplicity. The structure of $\beta$-FeOOH fundamentally considerably differs from that of $\alpha-\mathrm{FeOOH}$ and $\gamma-\mathrm{FeOOH}$, since $\beta$-FeOOH originally contains chloride ions, which are arranged in body centered cubic array of $\beta$-FeOOH. The structure of $\beta-\mathrm{FeOOH}$ consists of double chains of edge-shared $\mathrm{FeO}_{6}$ octahedral, and the double chains share corners with adjacent chains to give a three-dimensional structure containing tunnels. These tunnels run parallel to the $c$-axis, and they are considered to be stabilized by $\mathrm{Cl}^{-}$ions and the average number $1.23 \mathrm{Cl}^{-}$ ions per unit cell occupy two third of the tunnel. ${ }^{2)}$ Contrary to such ideal case, the realistic atomic arrangements of $\beta$ $\mathrm{FeOOH}$ and $\beta$-30S estimated by the RMC simulation technique are shown in Fig. 8(b) and (c). The atomic arrangements in $\beta$-FeOOH are similar to those of the ideal structure, while the atomic arrangements in $\beta$-30S are significantly distorted.

\subsection{Atomic-scale distortion observed in $\boldsymbol{\beta}$-FeOOH}

The distortion in the atomic-scale structure of $\beta-30 \mathrm{~S}$ is likely to arise from to the incorporation of sulfate ions in the $\beta$-FeOOH structure, which is suggested by FT-IR as shown in Fig. 1. Thus, anions are considered to influence the linkages of $\mathrm{FeO}_{6}$ structural units, together with cations. In this work, an influence of sulfur in X-ray scattering intensity was assumed to be small in calculation for the structure of $\beta$-30S. This is because the sulfur is relatively light elements in the present X-ray scattering measurements and atomic position of sulfur in the unit cell is not be specified. Along with such assumptions, heterogeneous distribution of cations, anions and impurities by addition of sulfate ions in $\beta$-FeOOH particles may cause some difference between the computed atomic-scale arrangement and the real structure. Nevertheless, the atomic-scale structure for $\beta$-FeOOH and $\beta$-30S estimated in this work appears to be reasonable, because they were calculated from the independent information obtained by ordinary and anomalous X-ray scattering measurements.

In addition, the interaction between cations and anions, (a)

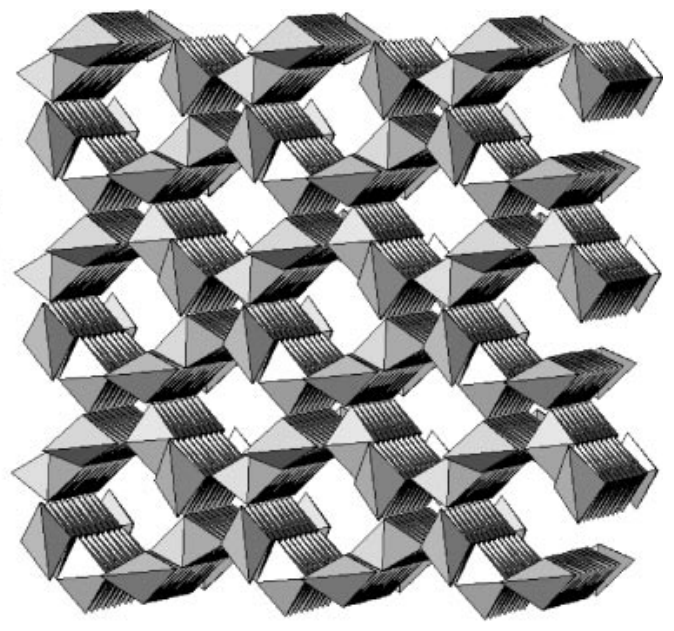

(b)

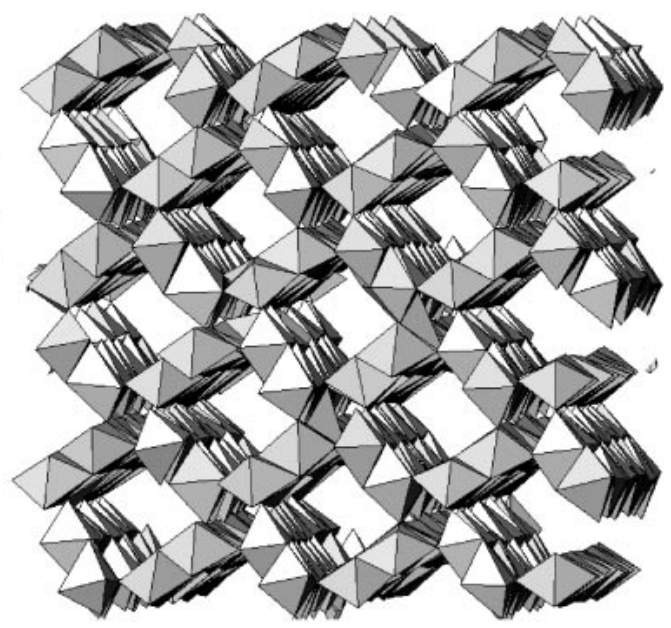

(c)

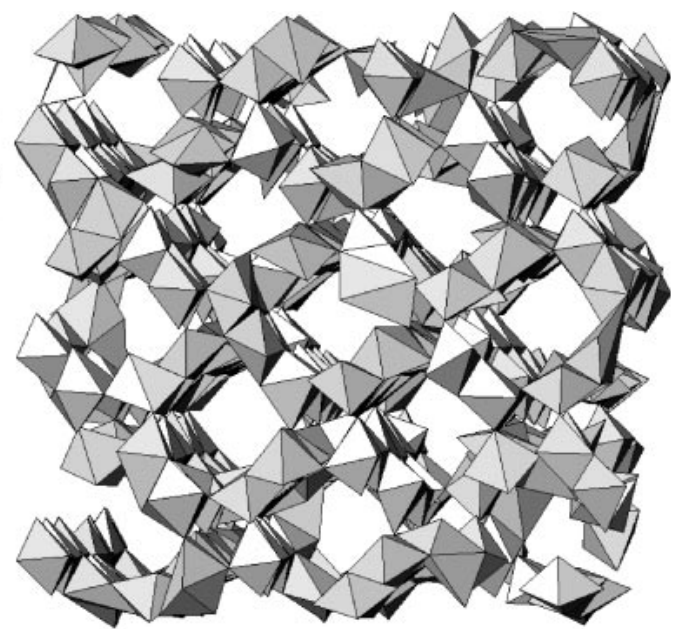

Fig. 8 Atomic arrangements of (a) the ideal $\beta$-FeOOH structure, (b) $\beta$ $\mathrm{FeOOH}$ and (c) $\beta$-30S using the $\mathrm{FeO}_{6}$ octahedral structural units estimated by RMC simulation.

which are incorporated to ferric oxyhydroxides, should be discussed, since cations as well as anions are likely to influence the atomic-scale structure of ferric oxyhydroxides. For instance, chromium sulfates are sometime added to aqueous solution containing ferric ions in synthesis of $\alpha$-FeOOH and $\beta$-FeOOH containing chromium, ${ }^{8,37)}$ in which sulfate ions may be interacted with chromium. The distortion 
in the atomic-scale structure of $\beta$-FeOOH may be enhanced by the incorporation of sulfate and chromium ions because of the mutual interaction between chromium and sulfate ions. ${ }^{38)}$ Thus, foreign cations and anions are considered to play an important role in the formation of ferric oxyhydroxides through co-precipitation and adsorption of the foreign ions in a complicated manner.

\section{Conclusions}

Quantitative X-ray structural analysis by applying anomalous X-ray scattering, coupled with the reverse Monte Carlo (RMC) simulation and Fourier-transform infrared spectroscopy, were used for characterizing the influence of sulfate ions on the atomic-scale structure of $\beta$-FeOOH. The main conclusions are as follows:

(1) Infrared spectra of $\beta$-FeOOH containing sulfate ions revealed new absorption bands appeared, which result presumably from the incorporation of sulfate ions to the solid particles.

(2) X-ray diffraction profiles showed that the structure of $\beta$ $\mathrm{FeOOH}$ is distorted by the incorporation of sulfate ions in it, although the structure is fundamentally similar to that of pure $\beta$-FeOOH.

(3) The pair distribution functions and atomic arrangements estimated by RMC simulation indicated that the fundamental structures of $\mathrm{FeO}_{6}$ octahedral units are not influenced severely by addition of sulfate ions, but the linkages of $\mathrm{FeO}_{6}$ octahedral units in $\beta$-FeOOH are considerably distorted by the incorporation of sulfate ions.

\section{Acknowledgements}

The present authors wish to express their thanks to Prof. M. Nomura, Photon Factory, High Energy Accelerator Research Organization located at Tsukuba in Japan, for his help on AXS measurements (Proposal No. 2003G182). The authors would also like to express gratitude to Prof. E. Matsubara and Prof. K. Sugiyama for their valuable discussion with the authors. This work was supported by the Grant-in-Aid for Scientific Research Fund from the Japan Society for Promotion of Science (No. 17206075).

\section{REFERENCES}

1) C. Leygraf and T. Graedel: Atmospheric Corrosion, (Wiley-Interscience, New York 2000) pp. 9-24.

2) R. M. Cornell and U. Schwertmann: The Iron Oxides, (Wiley-VCH, Weinheim 2003) pp. 185-199.

3) J. P. Jolivet, M. Henry, J. Livage and E. Bescher: Metal Oxide Chemistry and Synthesis, (Wiley-VCH, Chichester 1994) pp. 1-487.

4) J. P. Jolivet, C. Chaneac and C. Fiaud: Chem. Commun. (2004) 481487.

5) T. Sugimoto, K. Sakata and A. Muramatsu: J. Colloid Interface Sci. 159 (1993) 372-382.
6) K. Shinoda, E. Matsubara, A. Muramatsu and Y. Waseda: Mater. Trans. JIM 35 (1994) 394-398.

7) S. Suzuki, M. Saito, M. Kimura, T. Suzuki, H. Kihira and Y. Waseda: ISIJ International 43 (2003) 366-372.

8) S. Suzuki, Y. Takahashi, M. Saito, M. Kusakabe, T. Kamimura, H. Miyuki and Y. Waseda: Corros. Sci. 47 (2005) 1271-1284.

9) S. K. Kwon, S. Suzuki, M. Saito and Y. Waseda: Corros. Sci. (2005), in press.

10) T. Ishikawa, R. Katoh, A. Yasukawa, K. Kandori, T. Nakayama and F. Yuse: Corros. Sci. 43 (2001) 1727-1738.

11) T. Ishikawa, T. Motoki, R. Katoh, A. Yasukawa, K. Kandori, T. Nakayama and F. Yuse: J. Colloid Interface Sci. 250 (2002) 74-81.

12) K. Kanie, A. Muramatsu, S. Suzuki and Y. Waseda: Mater. Trans. 45 (2004) 968-971.

13) S. K. Kwon, K. Kimijima, K. Kanie, A. Muramatsu, S. Suzuki, E. Matsubara and Y. Waseda: ISIJ International 45 (2005) 77-81.

14) S. K. Kwon, K. Kimijima, K. Kanie, A. Muramatsu, S. Suzuki, E. Matsubara and Y. Waseda: Mater. Trans. 46 (2005) 155-158.

15) S. Suzuki, T. Suzuki, M. Kimura, Y. Takagi, K. Shinoda, K. Tohji and Y. Waseda: Appl. Surf. Sci. 169 (2001) 109-112.

16) M. Yamashita, T. Shimizu, H. Konishi, J. Mizuki and H. Uchida: Corros. Sci. 45 (2003) 381-394.

17) S. Suzuki, Y. Takahashi, T. Kamimura, H. Miyuki, K. Shinoda, K. Tohji and Y. Waseda: Corros. Sci. 46 (2004) 1751-1763.

18) P. A. Lee, P. H. Citrin, P. Eisenberger and B. M. Kincaid: Rev. Mod. Phys. 53 (1981) 769-806.

19) B. K. Teo and P. A. Lee: J. Am. Chem. Soc. 99 (1977) 3856-3859.

20) Y. Waseda: Anomalous X-Ray Scattering for Materials Characterization, (Springer-Verlag, Heidelberg 2002) pp. 21-38.

21) H. D. Ruan, R. L. Frost, J. T. Kloprogge and L. Duong: Spectrochim. Acta Part A 58 (2002) 967-981.

22) N. Boucherit, A. Hugot-Le Goff and S. Joiret: Corros. Sci. 32 (1991) 497-507.

$23)$ D. Thierry, D. Persson, C. Leygraf, N. Boucherit and A. Hugot-Le Goff: Corros. Sci. 32 (1991) 273-284.

24) S. Nasu, T. Kamimura and T. Tazaki: Hyp. Interact. 139 (2002) 175182.

25) T. Kamimura, S. Nasu, T. Segi, T. Tazaki, S. Morimoto and H. Miyuki: Corros. Sci. 45 (2003) 1863-1879.

26) M. Saito, S. Kang and Y. Waseda: Jpn J. Appl. Phys. 38 (1999) 596599.

27) M. Saito, S. Kang and Y. Waseda: J. Phys. Sci. Jpn. 68 (1999) 19321938.

28) S. Kang, C. Park, M. Saito and Y. Waseda: Mater. Trans. JIM 40 (1999) $552-555$.

29) A. Muramatsu: Morphology Control of Materials and Nanoparticles, eds. by Y. Waseda and A. Muramatsu, (Springer-Verlag, Heidelberg 2003) pp. 25-62.

30) T. Sugimoto, M. M. Kahn, K. Sakata, A. Muramatsu and H. Itoh: Colloids Surf. A 79 (1993) 233-247.

31) T. Sugimoto, Y. Wang, H. Itoh and A. Muramatsu: Colloids Surf. A 134 (1998) 265-279.

32) D. Shindo, G. S. Park, Y. Waseda and T. Sugimoto: J. Colloid Interface Sci. 168 (1994) 478-484.

33) T. Sugimoto, S. Waki, H. Itoh and A. Muramatsu: Colloids Surf. A 109 (1996) 155-165.

34) T. Sugimoto and A. Muramatsu: J. Colloid Interface Sci. 184 (1996) 626-638.

35) T. Sugimoto and Y. Wang: J. Colloid Interface Sci. 207 (1998) 137149.

36) J. M. Bigham, U. Schwertmann, L. Carlson and E. Murad: Geochim. Cosmochim. Acta 54 (1990) 2743-2758.

37) T. Kamimura, S. Nasu, T. Segi, T. Tazaki, H. Miyuki, S. Morimoto and T. Kudo: Corros. Sci. (2005), in press.

38) S. K. Kwon, S. Suzuki, M. Saito, T. Kamimura, H. Miyuki and Y. Waseda: Corros. Sci., in press. 